Beurs, D. de, Kirtley, O., Kerkhof, A., Portzky, G., O'Connor, R.C. The role of mobile phone technology in understanding and preventing suicidal behavior. Crisis: The Journal of Crisis Intervention and Suicide Prevention: 2015, 36(2), 79-82

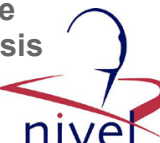

\begin{tabular}{|l|l|}
\hline $\begin{array}{l}\text { Postprint } \\
\text { Version }\end{array}$ & 1.0 \\
\hline Journal website & http://psycnet.apa.org/journals/cri/36/2/79/ \\
\hline Pubmed link & \\
\hline DOI & $10.1027 / 0227-5910 / a 000316$ \\
\hline
\end{tabular}

This is a NIVEL certified Post Print, more info at http://www.nivel.eu

\title{
The Role of Mobile Phone Technology in Understanding and Preventing Suicidal Behavior
}

\author{
De Beurs, DereK; Kirtley, Olivia; KerkHoF, AD; PortzKy, GWENDOlyn; O’CONNOR, \\ RORY C.
}

Netherlands Institute for Health Services Research (Nivel), Utrecht, The Netherlands Suicidal Behaviour Research Laboratory, University of Glasgow, UK

Department of Clinical Psychology, Vrije Universiteit Amsterdam, The Netherlands Unit for Suicide Research, Ghent University, Belgium

Derek de Beurs Netherlands Institute for Health Services Research (Nivel)

Suicidal behavior is the result of a complex interaction of psychiatric, psychological, social, and cultural factors (Hawton \& van Heeringen, 2009; O'Connor \& Nock, 2014; O’Connor, Platt, \& Gordon, 2011b). Despite considerable epidemiological advances in the identification of risk factors, explanations (as distinct from risk factors) for why suicide occurs in some cases but not in others are limited. In addition, the factors that predict the transition from suicidal thoughts to suicidal behavior are poorly understood and the evidence for which treatments are effective in reducing suicide risk is also scant. In this editorial, we discuss how mobile phone technology has the potential to move the field forward in terms of understanding suicide risk as well as laying foundations for the development of effective treatments/interventions. We have focused on mobile health technology given the rapid growth of mobile health approaches in suicide prevention (De Jaegere \& Portzky, 2014; Mishara \& Kerkhof, 2013) and psychological research more generally (Myin-Germeys et al., 2009; Nock, Prinstein, \& Sterba, 2009; Palmier-Claus et al., 2011) and because mobile phone use is ubiquitous, with $75 \%$ of the world having access to a mobile phone (Kay, 2011).

While we believe that health interventions delivered via mobile phones have the potential to lead to more promising and dynamic intervention and prevention strategies, in the following sections, we highlight the limitations and barriers to their use as well as their strengths and the opportunities that they afford.

\section{Understanding AND PREDICTION}

A myriad of prospective studies have tried to predict which individual patients will attempt suicide or die by suicide (Beck \& Steer, 1989; Brown, Beck, Steer, \& 
Beurs, D. de, Kirtley, O., Kerkhof, A., Portzky, G., O'Connor, R.C. The role of mobile phone technology in understanding and preventing suicidal behavior. Crisis: The Journal of Crisis Intervention and Suicide Prevention: 2015, 36(2), 79-82

Grisham, 2000; O’Connor, Smyth, Ferguson, Ryan, \& Williams, 2013; Riihimäki, Vuorilehto, Melartin, Haukka, \& Isometsä, 2014) and a range of risk factors that correlate with suicidal behavior have been identified. Nonetheless it remains difficult to predict with sufficient sensitivity/specificity which individuals who express suicidal thoughts will attempt suicide or die by suicide. Indeed, there are many reasons why it is difficult to predict the transition from suicidal thoughts to suicidal behavior; however, the fact that data in most studies are collected retrospectively at a single time point in a nonnatural setting (Nock et al., 2009; usually in a hospital or a university laboratory) is likely to be part of the problem. Data derived from studies of this kind are likely to be affected by respondent bias, and, importantly, such study designs do not take into account the fluctuating nature of suicidal thinking and urges to attempt suicide. Indeed, it is well established that suicidal thinking waxes and wanes (Joiner Jr. \& Rudd, 2000; Witte, Fitzpatrick, Joiner Jr., \& Schmidt, 2005). Given these challenges, collecting data in real time via mobile phones, employing ecological momentary assessment (EMA) techniques, offers considerable promise to better understand the transition from suicidal thoughts to behavior (Myin-Germeys et al., 2009; Nock et al., 2009; Palmier-Claus et al., 2011). EMA is defined as the realtime sampling of current behaviors and experiences in the natural environment (Moskowitz \& Young, 2006). A pioneering study by Nock et al. (2009) demonstrated the feasibility and potential of EMA in the understanding of suicidal behavior. They found, for example, that suicidal thoughts occurred less frequently than nonsuicidal self-injury thoughts. The technology to conduct real-time EMA data collection is widely available; by downloading an app onto the phone of patients, information about their thoughts and behavior can be collected in real-time via pop-up messages. Alternatively, data can be collected unobtrusively by mobile phone sensors or by collecting smart phone usage patterns (Likamwa, Liu, Lane, \& Zhong, 2013). For example, it is possible to combine unobtrusively collected data from SMS (short message service), e-mail, social media, and telephone usage, and use these data to accurately predict an individual's current mood (Likamwa et al., 2013). By analyzing the frequency of smartphone usage (without analyzing any content), the user's daily mood average could be estimated with an accuracy of 93\%. EMA data can also be analyzed using a novel methodology called network analysis (Borsboom \& Cramer, $\underline{2013}$ ). Network analysis differs from traditional statistical analysis in that it does not regard psychiatric symptoms as manifestations of an underlying disorder, but rather as elements within a dynamic network. From a network perspective, each suicidal patient has their own network of symptoms that may drive behavior. Consequently, the same levels of rumination and sleep disturbance, for example, that would result in suicidal behavior in one person might not result in suicidal behavior in someone else, because of individual differences in the interaction of symptoms. Via network analysis, based on EMA data, individual pathways to suicidal behavior can be determined. We believe that network analytic approaches afford a more fine-grained appreciation of the suicidal mind, and therefore they should usefully contribute to a refinement of the existing explanatory models of suicidal behavior.

Currently, most experimental studies rely on convenience samples, often students or patients from a nearby hospital, thereby limiting generalizability. By contrast, mobile phone studies can recruit participants more easily from more difficult-to-reach populations, from across the life span, from different ethnic backgrounds, and from 
Beurs, D. de, Kirtley, O., Kerkhof, A., Portzky, G., O'Connor, R.C. The role of mobile phone technology in understanding and preventing suicidal behavior. Crisis: The Journal of Crisis Intervention and Suicide Prevention: 2015, 36(2), 79-82

countries not commonly included in suicide research studies ( $\underline{\mathrm{O}^{\prime} \text { Connor, Platt, \& }}$ Gordon, 2011a).

\section{INTERVENTION AND PREVENTION}

Data collected via EMA can be used to offer personally tailored interventions (ecological momentary intervention $[\mathrm{EMI}]$ ) to individuals on their mobile device as they go about their daily lives (Heron \& Smyth, 2010; Runyan et al., 2013). To do so, EMA data are processed via carefully designed algorithms that combine all the data inputs which, in turn, inform the selection of the most appropriate intervention for a particular patient. Compared with nonmobile intervention studies, EMI-based studies can lead to larger and better powered clinical trials of psychological treatments to reduce suicide ideation, suicide attempts, and suicide deaths. EMI allows us to develop and test brief psychosocial interventions, centered on evidencebased interventions such as CBT (van Spijker, van Straten, \& Kerkhof, 2014) or a safety plan (Stanley \& Brown, 2012). Potentially, therapeutic intervention can be offered several times a day, instead of only once a week (Cuijpers, Huibers, Daniel Ebert, Koole, \& Andersson, 2013). Furthermore, the wide availability of mobile phones may help to reach individuals who are not reached by traditional services, such as adolescents and young adults (Asarnow \& Miranda, 2014). For example, studies (Ravert, Calix, \& Sullivan, 2010; Runyan et al., 2013) have already demonstrated the feasibility and potential effectiveness of mobile health apps for undergraduate students. Importantly, as mobile phones are becoming more widely available in low- and middle-income countries (James, 2014), in the future EMA/EMI might allow us to develop, offer, and test interventions specifically tailored for populations that lack adequate infrastructure and resources for mental health care.

\section{BARRIERS AND LIMITATIONS}

Several barriers and limitations should be taken into account when using mobile health technologies to understand and prevent suicide.

\section{Ethical Considerations}

Privacy and data security are of great concern when using mobile health methods (Brian \& Ben-Zeev, 2014; De Jaegere \& Portzky, 2014). EMA data, like all personal data, are very sensitive and should, therefore, be collected and treated with the utmost care. Importantly, the user should be actively informed about potential security risks, which calls for very clear informed consent, with full disclosure of all information that a potential user needs to make a decision about using the app (De Jaegere \& Portzky, 2014). Also, using an app among vulnerable participants calls for a clear crisis intervention plan that describes how suicidal crises will be dealt with when they arise. Finally, adequate testing of the appropriateness of the EMA/EMI program for different suicidal target groups and even prescreening individuals before the use of the app are important to ensure suitability of the program for potential participants. Alternatives (such as suicide crisis lines) should be widely available and clearly offered if the app is not the correct intervention for the user (De Jaegere \& Portzky, 2014). As privacy and data security are under current debate (Kotz, 2011), future researchers should realize that ethics committees and institutional review boards will not easily approve mobile health-based research on suicidal behavior 
Beurs, D. de, Kirtley, O., Kerkhof, A., Portzky, G., O'Connor, R.C. The role of mobile phone technology in understanding and preventing suicidal behavior. Crisis: The Journal of Crisis Intervention and Suicide Prevention: 2015, 36(2), 79-82

without full and thorough consideration of both the safety of the participants and their data.

\section{Multidisciplinary Team}

We emphasize the necessity for multidisciplinary collaborations when using mobile phone technology for psychological research. For one, programming and designing a good app is challenging and time-consuming. The apps should work fast, without any bugs, and be able to function on different operating systems (e.g., iOS, Android, Windows). A further and often neglected consideration is the app has to be well designed and user friendly; ease of use could be a crucial factor in maintaining participants' use of the app. Furthermore, big data require complex statistical methods that are not well suited to standard statistical software such as SPSS, with many previous EMA/EMI studies employing multilevel modeling analyses using R, SAS, or MPlus (Armey, Crowther, \& Miller, 2011). Computer modeling is also required for the reasoning model (e.g., if $\mathrm{x}$, then $\mathrm{y}$ ) that underpins automated EMI. In sum, to use new technologies effectively in psychological research, one ideally needs a team of health scientists, (bio) statisticians, programmers, designers, and computer scientists. As all disciplines have their own scientific language and work process, time should be taken to develop a constructive working alliance.

\section{LIMITATIONS}

As promising as mobile phone technologies may sound, it is also important to be realistic. EMA/EMI is not the ultimate panacea; it will not solve all problems of measurement, understanding, and prevention within suicidology. EMA and subsequent network analysis will not lead to a complete understanding of the transition of suicidal thoughts to suicidal behavior or to a flawless prediction model of death by suicide. Moreover, a substantial part of the target group will still not be reached by mobile interventions, no matter how well designed the app is. Offering mobile phone interventions in low- and middle-income countries sounds promising, but currently it will be difficult to realize due to information and communication technology barriers such as the quality of the telecommunication network and limited access to power supplies (Brian \& Ben-Zeev, 2014).

\section{IN SUM}

Mobile phone technologies offer a unique chance to improve our understanding of the antecedents of suicidal behavior. In particular, they allow us to focus on the temporal transition from suicidal thoughts to suicidal behaviors; something about which we still know little. Tailored interventions based on mobile data and offered via a mobile device might improve our current intervention toolkit, and the widespread availability of mobile phones allows us to reach new groups, unreachable by nonmobile public health interventions. However, one should be realistic and reflect on the various challenges and limitations of these methods. Mobile health should not be regarded as the solution for every suicidal individual, but as a promising new direction that can lead to new insights and better understanding of suicidal thoughts and behaviors. 
Beurs, D. de, Kirtley, O., Kerkhof, A., Portzky, G., O'Connor, R.C. The role of mobile phone technology in understanding and preventing suicidal behavior. Crisis: The Journal of Crisis Intervention and Suicide Prevention: 2015, 36(2), 79-82

\section{REFERENCES}

Armey M. F., Crowther J. H., Miller I. W. (2011). Changes in ecological momentary assessment reported affect associated with episodes of nonsuicidal self-injury. Behaviour Therapy, 42(4), 579-588.

Asarnow J. R., Miranda J. (2014). Improving care for depression and suicide risk in adolescents: Innovative strategies for bringing treatments to community settings. Annual Review of Clinical Psychology, 10, 275-303.

Beck A. T., Steer R. A. (1989). Clinical predictors of eventual suicide: A 5-to 10year prospective study of suicide attempters. Journal of Affective Disorders, 17(3), 203-209.

Borsboom D., Cramer A. O. (2013). Network analysis: An integrative approach to the structure of psychopathology. Annual Review of Clinical Psychology, 9, 91-121.

Brian R. M., Ben-Zeev D. (2014). Mobile health (mHealth) for mental health in Asia: Objectives, strategies, and limitations. Asian Journal of Psychiatry, 10, 96-100

Brown G. K., Beck A. T., Steer R. A., Grisham J. R. (2000). Risk factors for suicide in psychiatric outpatients: A 20-year prospective study. Journal of Consulting and Clinical Psychology, 68(3), 371.

Cuijpers P., Huibers M., Daniel Ebert D., Koole S. L., Andersson G. (2013). How much psychotherapy is needed to treat depression? A metaregression analysis. Journal of Affective Disorders, 149(1), 1-13.

De Jaegere E., Portzky G. (2014). Analysis of available technology-based suicide prevention programmes. Retrieved from http://www.euregenas.eu/wpcontent/uploads/2014/03/Analysis-of-TBSP-programmes.pdf

Hawton K., van Heeringen K. (2009). Suicide. The Lancet, 373(9672), 1372-1381.

Heron K. E., Smyth J. M. (2010). Ecological momentary interventions: Incorporating mobile technology into psychosocial and health behaviour treatments. British Journal of Health Psychology, 15(1), 1-39.

James J. (2014). Relative and absolute components of leapfrogging in mobile phones by developing countries. Telematics and Informatics, 31(1), 52-61.

Joiner T. E. Jr., Rudd M. D. (2000). Intensity and duration of suicidal crisis vary as a function of previous suicide attempts and negative life events. Journal of Consulting and Clinical Psychology, 68(5), 909.

Kay M. (2011, ). mHealth: New horizons for health through mobile technologies. Paper presented at the GSMA mHA Mobile Health Summit, Cape Town, South Africa. Retrieved form http://www.who.int/ehealth/mhealth summit.pdf 
Beurs, D. de, Kirtley, O., Kerkhof, A., Portzky, G., O'Connor, R.C. The role of mobile phone technology in understanding and preventing suicidal behavior. Crisis: The Journal of Crisis Intervention and Suicide Prevention: 2015, 36(2), 79-82

Kotz D. (2011, ). A threat taxonomy for mHealth privacy. Paper presented at the Third Conference on Communication Systems and Networks,Bangalore.

Likamwa R. L., Liu Y., Lane N. D., Zhong L. (2013, ). MoodScope: Building a mood sensor from smartphone usage patterns. Paper presented at the International Conference on Mobile Systems, Applications and Services, Taiwan.

Mishara B., Kerkhof A. (2013). Suicide prevention and new technologies: Evidence based practice. Basingstoke, UK: Palgrave Macmillan.

Moskowitz D. S., Young S. N. (2006). Ecological momentary assessment: What it is and why it is a method of the future in clinical psychopharmacology. Journal of Psychiatry and Neuroscience, 31(1), 13.

Myin-Germeys I., Oorschot M., Collip D., Lataster J., Delespaul P., van Os J. (2009). Experience sampling research in psychopathology: Opening the black box of daily life. Psychological Medicine, 39(09), 1533-1547.

Nock M. K., Prinstein M. J., Sterba S. K. (2009). Revealing the form and function of self-injurious thoughts and behaviours: A real-time ecological assessment study among adolescents and young adults. Journal of Abnormal Psychology, 118(4), 816.

O’Connor R. C., Nock M. K. (2014). The psychology of suicidal behaviour. The Lancet Psychiatry, 1(1), 73-85.

O’Connor R. C., Platt S., Gordon J. (2011a). Achievements and challenges in suicidology: Conclusions and future directions. In R. C. O'Connor, S. Platt, J. Gordon (), International handbook of suicide prevention: Research, policy and practice (pp. 625-642). Chichester, UK: John Wiley \& Sons

O’Connor R. C., Platt S., Gordon J. (). (2011b). International handbook of suicide prevention: Research, policy and practice. Chichester, UK: John Wiley \& Sons.

O’Connor R. C., Smyth R., Ferguson E., Ryan C., Williams J. M. G. (2013). Psychological processes and repeat suicidal behaviour: A four-year prospective study. Journal of Consulting and Clinical Psychology, 81(6), 1137-1143.

Palmier-Claus J., Myin-Germeys I., Barkus E., Bentley L., Udachina A., Delespaul P., Dunn G., et al. (2011). Experience sampling research in individuals with mental illness: Reflections and guidance. Acta Psychiatrica Scandinavica, 123(1), 12-20.

Ravert R. D., Calix S. I., Sullivan M. J. (2010). Research in brief: Using mobile phones to collect daily experience data from college undergraduates. Journal of College Student Development, 51(3), 343-352.

Riihimäki K., Vuorilehto M., Melartin T., Haukka J., Isometsä E. (2014). Incidence and predictors of suicide attempts among primary-care patients with depressive disorders: A 5-year prospective study. Psychological Medicine, 44(02), 291-302. 
Beurs, D. de, Kirtley, O., Kerkhof, A., Portzky, G., O'Connor, R.C. The role of mobile phone technology in understanding and preventing suicidal behavior. Crisis: The Journal of Crisis Intervention and Suicide Prevention: 2015, 36(2), 79-82

Runyan J. D., Steenbergh T. A., Bainbridge C., Daugherty D. A., Oke L., Fry B. N. (2013). A smartphone ecological momentary assessment/intervention "app" for collecting real-time data and promoting self-awareness. PloS One, 8(8), e71325.

Stanley B., Brown G. K. (2012). Safety planning intervention: A brief intervention to mitigate suicide risk. Cognitive and Behavioural Practice, 19(2), 256-264.

van Spijker B. A., van Straten A., Kerkhof A. J. (2014). Effectiveness of online selfhelp for suicidal thoughts: Results of a randomised controlled trial. PloS One, 9(2), e90118.

Witte T. K., Fitzpatrick K. K., Joiner T. E. Jr., Schmidt N. B. (2005). Variability in suicidal ideation: A better predictor of suicide attempts than intensity or duration of ideation? Journal of Affective Disorders, 88(2), 131-136. 
Beurs, D. de, Kirtley, O., Kerkhof, A., Portzky, G., O'Connor, R.C. The role of mobile phone technology in understanding and preventing suicidal behavior. Crisis: The Journal of Crisis Intervention and Suicide Prevention: 2015, 36(2), 79-82 